\title{
An Overview of Polarimetric Sensing Techniques and Technology with Applications to Different Research Fields
}

\author{
Frans Snik ${ }^{a}$, Julia Craven-Jones ${ }^{b}$, Michael Escuti $^{c}$, Silvano Fineschi ${ }^{d}$, David Harrington ${ }^{e}$, \\ Antonello de Martino ${ }^{f}$, Dimitri Mawet ${ }^{g}$, Jérôme Riedi ${ }^{h}$, J. Scott Tyo ${ }^{i}$

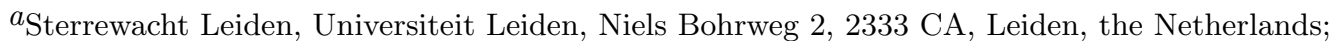

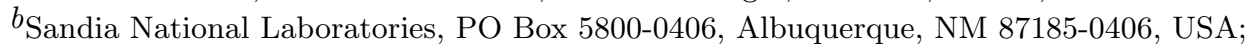 \\ ${ }^{c}$ North Carolina State University, 432 Monteith, Campus Box 7914, Raleigh, NC 27695-7914, USA;

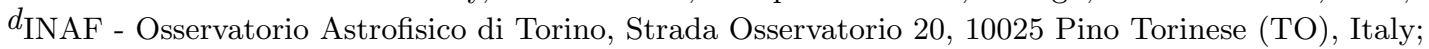 \\ ${ }^{e}$ University of Hawaii, Institute for Astronomy, 2680 Woodlawn Drive, Honolulu, HI 96822-1839, USA;

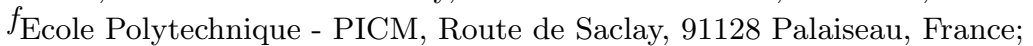

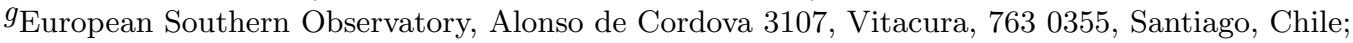

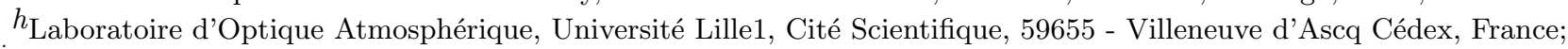 \\ ${ }^{i}$ College of Optical Sciences, Univ. of Arizona, 1630 E. University Blvd., PO Box 210094, Tucson, AZ 85721-0094, USA.
}

\begin{abstract}
We report the main conclusions from an interactive, multidisciplinary workshop on "Polarimetric Techniques and Technology", held on March 24-28 2014 at the Lorentz Center in Leiden, the Netherlands. The workshop brought together polarimetrists from different research fields. Participants had backgrounds ranging from academia to industrial R\&D. Here we provide an overview of polarimetric instrumentation in the optical regime geared towards a wide range of applications: atmospheric remote sensing, target detection, astronomy, biomedical applications, etc. We identify common approaches and challenges. We list novel polarimetric techniques and polarization technologies that enable promising new solutions. We conclude with recommendations to the polarimetric community at large on joint efforts for exchanging expertise.
\end{abstract}

Keywords: polarimetry

\section{INTRODUCTION}

Polarization is a fundamental property of light, and light from any source is polarized to some degree. Polarimetry is therefore a valuable technique to remotely obtain information about a wide range of sources and objects. It is therefore implemented in many different research fields, in many different ways. This paper reviews those implementations of polarimetry and their applications. Furthermore, we identify common challenges for polarimetric applications, and we list new developments and novel techniques that are of general benefit. As polarimetry is still evolving as a general-purpose technique, and often considered specialistic and difficult, we aim to provide an overview to the non-specialist reader. We refer to recent books, review papers and landmark publications wherever possible.

This publication is one of the results from an interactive workshop on "Polarimetric Techniques \& Technology", organized at the Lorentz Center* in Leiden, the Netherlands. This workshop was co-organized by the EU COST network "Polarisation as a tool to study the solar system and beyond" $\dagger$. The authors of this paper acted as the Scientific Organizing Committee. This workshop brought together polarimetrists from a wide range of scientific backgrounds (atmospheric remote sensing, target detection, astronomy, biomedical applications, etc.), as well as representatives from industry. The first two days of the workshop consisted of contributed talks to inform the audience about the state-of-the-art and challenges of polarimetric applications, whereas the remaining three days were dedicated to plenary and splinter discussion sessions and interactive design challenges. This paper reports on the most notable outcomes of these talks, discussions and assignments.

email: snik@strw.leidenuniv.nl

*www.lorentzcenter.nl

${ }^{\dagger}$ www.polarisation.eu

Polarization: Measurement, Analysis, and Remote Sensing XI, edited by David B. Chenault, Dennis H. Goldstein, Proc. of SPIE Vol. 9099, 90990B - (C) 2014 SPIE · CCC code: 0277-786X/14/\$18 · doi: 10.1117/12.2053245 


\section{CONVENTIONS}

All fields of optical (i.e. the UV-IR range) polarimetry adopt the Stokes formalism to describe the polarization measurement, as the detectors are only sensitive to the total intensity (photon count) and not to phase and amplitude of the incident light, or the orientation of the electric field. Moreover, the Stokes formalism is ideally suited for the description of partially polarized light, and light in nature is almost never fully polarized. The operational definition of the Stokes vector conveniently describe the polarized Stokes parameters as differences of intensity measurements. The second and third Stokes parameters fully describe the linear polarization state of light, and the fourth one describes circular polarization. In many cases, it is preferable to describe the measurement results in terms of fractional polarization (by dividing the polarized Stokes parameters by the intensity), or in terms of degree of (linear/circular) polarization and angle of (linear) polarization.

While the same mathematic language is spoken in the separate communities, several dialects are prevalent. For instance, the remote sensing / target detection community denotes the Stokes parameters with $\left[S_{0}, S_{1}, S_{2}, S_{3}\right]^{T}{ }^{1}$ whereas the astronomical / atmospheric community uses $[I, Q, U, V]^{T}{ }^{2}$. While the first convention is convenient for index arithmetic in vector/matrix calculus, it can also be confusing as in many fields of optics $S_{i}$ is adopted, e.g., to list measured signals. The use of $[I, Q, U, V]^{T}$ has a historical origin, as this is the notation that Chandrasekhar used when he reintroduced the work of Stokes in 1947.

In the different fields, many sign conventions are in use for the coordinate system of $\left[S_{1}, S_{2}\right],[Q, U]$ and for the handedness of $S_{3}, V$, and in many cases these conventions are not even observed. For instance, the definitions of the IEEE and the International Astronomical Union ${ }^{3}$ are opposite to those that emerge from a basis of Pauli spin matrices. ${ }^{4}$ It is probably impossible to agree on a single convention, but it is apropos to emphasize here that authors should state their coordinate system for the Stokes parameters. In the end, it might only come down to a minus sign flip for $S_{2}, U$ and/or $S_{3}, V$, but this may still be crucial for the interpretation of the results.

Coherence effects cannot be described by the Stokes formalism, and are generally ignored. Nevertheless, in some cases interference effects are significant, and other formalisms need to be used: the Jones formalism, the Wolf coherence matrix formalism, ${ }^{5}$ and Berreman calculus ${ }^{6}$ (for thin-film interference effects). When using the Stokes formalism in scattering application with coherent illumination, i.e. in laser Mueller matrix polarimetry, care must be taken to properly account for the coherent effects of speckles in analyzing the data. ${ }^{7}$

Many different terms are in use to describe the quality of a polarimetric measurements: polarimetric "error", "noise", "sensitivity", "precision", "accuracy", "uncertainty", etc. The most well-defined terms are probably the "polarimetric sensitivity", which describes the noise level in fractional polarization above which a polarization signal can be detected, and the "polarimetric accuracy" that relates the measured Stokes parameters (after calibration) to the true ones. ${ }^{2,4}$ The latter should then be expressed as a $4 \times 4$ matrix in the case of a full-Stokes measurement, but it can often be compressed into a requirement for the polarization zero-point, the scale, and the cross-talk between linear and circular polarization.

\section{POLARIMETRIC APPLICATIONS}

\subsection{Atmospheric Remote Sensing}

Polarimetry is considered a crucial technique in atmospheric remote sensing, and, in particular, for characterization of aerosol particles. In fact, only the combination of polarization measurements of scattered sunlight with multi-spectral and multi-angle functionality allows for the unambiguous, remote retrieval of several aerosol properties: the aerosol optical thickness, and microphysical properties like the size distribution, the chemical composition (through the complex refractive index) and the particle shape (spherical, non-spherical, jagged, etc.). Such measurements are not only required to assess the health hazards of aerosols and to probe volcanic ash clouds that impact air traffic, they are also crucial to measure aerosol scattering/absorption properties that currently constitute the largest source of uncertainty within climate modeling. In addition to dedicated ground-based polarimetric instrumentation, ${ }^{9}$ there are several satellite instruments under development to provide polarimetric aerosol measurements on a global scale. We review these instruments, as they are also exemplary for the wide range of design options for polarimetric instrumentation.

The POLDER instrument ${ }^{10}$ has flown in three different incarnations and has pioneered space-based polarimetric remote sensing. Its design is also the blueprint for future, more powerful instruments, like 3MI onboard

Proc. of SPIE Vol. 9099 90990B-2 


\section{Intensity}
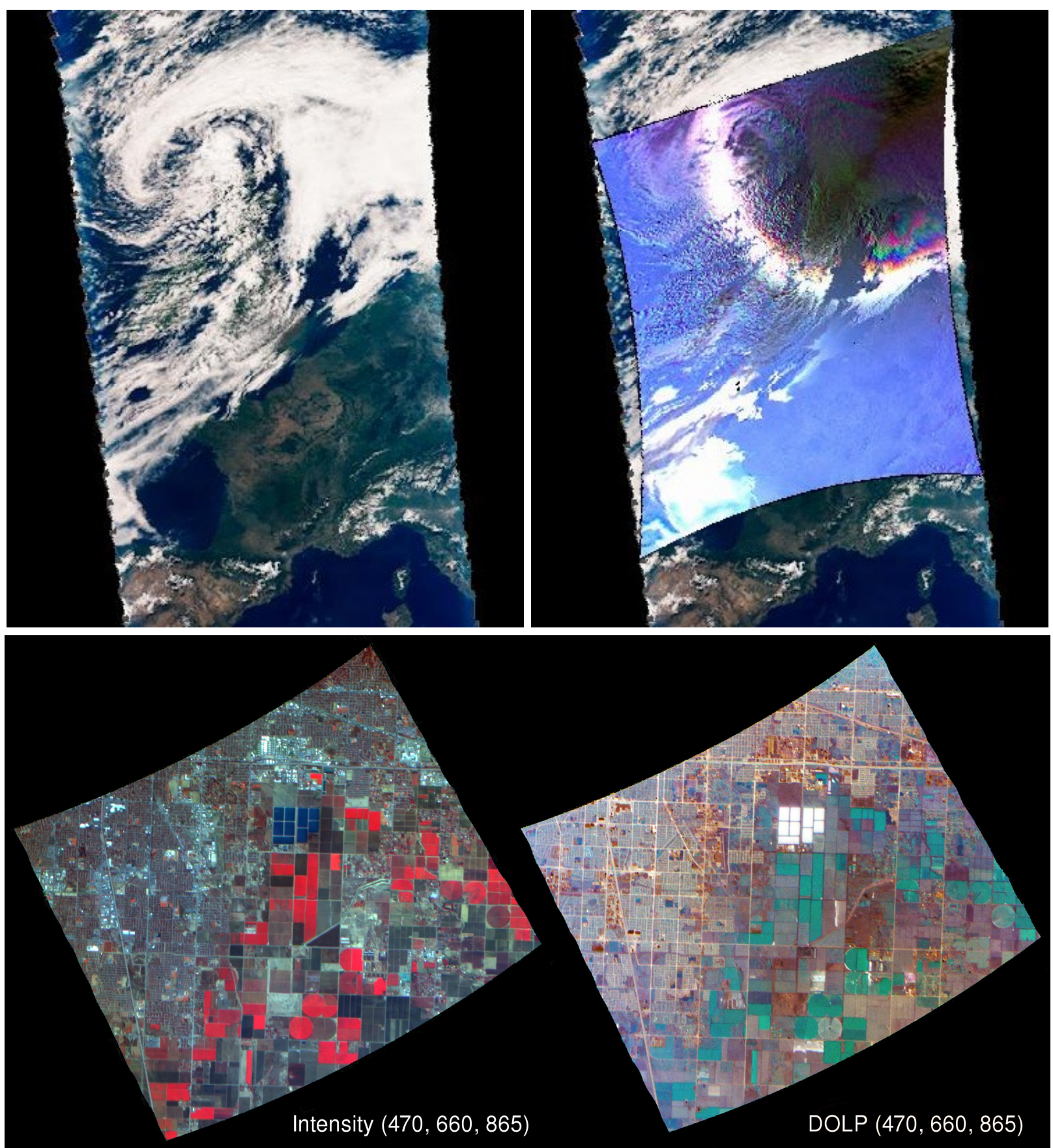

Figure 1. a) POLDER data: true-color intensity and corresponding polarized intensity at 440, 670 , 865 nm, exhibiting scattering properties of the atmosphere and cloudbow features that are used to retrieve droplet size (courtesy: Jérôme Riedi). b) MSPI data from PODEX $^{8}$ campaign, flying over Bakersfield, CA. Crop fields are clearly discerned, as are wastewater treatment ponds (courtesy: David J. Diner and the AirMSPI Team/Jet Propulsion Laboratory).

EPS-SG (EUMETSAT Polar System - Second Generation). POLDER is a fisheye imager that employs a filter wheel for its multi-spectral and also its multi-polarization capabilities. Several of the wavelength filters (433, $670,865 \mathrm{~nm}$ ) are repeated three times and paired with linear polarizers at 0, 60 and 120 degrees, such that the 
linear Stokes parameters can be measured for each of the filter bands after three positions of the filter wheel. As this filter wheel rotates while the satellite is flying over ground scenes, the three recordings differ slightly. After careful calibration (also of the instrumental polarization due to the fisheye optical system), the polarimetric accuracy for POLDER measurements is $\sim 2 \%$ for scenes with large spatial gradients. A sample of POLDER data is presented in Fig. 1a.

To provide more information about the chemical composition of aerosol particles, a polarimetric accuracy of $\sim 10^{-3}$ is required. Three, very different, polarimetric instrument concepts are being developed to provide such high-accuracy polarimetry. The Research Scanning Polarimeter ${ }^{11}$ and the Aerosol Polarimetry Sensor ${ }^{12}$ were designed to deliver strictly simultaneous linear polarization measurements. Unfortunately, the GLORY satellite that carried the APS instrument failed to achieve orbit in 2011. The polarimetry is implemented through several boresighted telescopes with dichroic beam-splitter and Wollaston prisms under $0 / 90^{\circ}$ and $\pm 45^{\circ}$ that feed multiple photodiodes. The ground scene is rapidly scanned by a rotating assembly of two crossed mirrors, such that their combined instrumental polarization properties are minimized. This mechanism can also be pointed to feed light through a polarization calibration unit that verifies the polarization accuracy of $\sim 0.2 \%$ that was achieved on-ground.

The Multi-angle SpectroPolarimeter Imager $\left(\mathrm{MSPI}^{13}\right)$ contains a three-mirror anastigmat telescope that images a strip of \pm 15 degrees along the flight direction onto 13 line detector strips that cover various wavelength bands. Observations under various angles along track are effectuated by sweeping a single instrument or by either employing a multitude of identical subsystems under different angles or scanning a single system back and forth. The polarization modulator is a dual photo-elastic modulator (PEM) system in between two athermal quarter-wave plates that are optimized for performance in the three polarimetric bands $(470,660,865 \mathrm{~nm})$. The beat frequency of the two PEMs at $12.5 \mathrm{~Hz}$ produces a modulation signal for the detector which is rapid enough to suppress polarization artifacts due to motion smearing down to the $\sim 0.5 \%$ level. The detector strips for the polarimetric wavelength bands are covered by wire-grid polarizers at 0 and 45 degrees, such that the linear Stokes parameters are measured simultaneously. The modulator package is located after the first two telescope mirrors, which are covered with low-polarization $(<1 \%)$ coatings. A sample image from the AirMSPI instrument is presented in Fig. 1b.

The SPEX concept ${ }^{14}$ is based on spectral polarization modulation (see subsection 4.1): a modulator package implemented for each viewing direction produces a sinusoidal modulation onto the spectrum for which the amplitude scales with the degree of linear polarization, and the phase is determined by the angle of linear polarization. The major advantage of this polarimetric technique is that all information on both spectrum and polarization is contained in a single shot, which renders it impervious to differential effects that often plague temporal or spatial modulation polarimeters. A polarimetric accuracy of $0.2 \%$ has been shown in the lab. Moreover, the spectral modulator can be located all the way upstream in the optical train, such that the instrumental polarization is minimal. The SPEX instrument employs a spectrometer that combines the (spectrally modulated) light from various viewing directions with extended swath onto a single slit. By locating a Wollaston prism in front of the objective lens for each viewing direction, two split spectra are imaged onto the slit, which contain opposite spectral polarization modulations, such that the sum of the two beams (after correction of transmission differences) yields the unmodulated intensity spectrum at full spectral resolution.

\subsection{Target Detection}

In target detection applications (e.g. machine vision, military application) polarimetry is often applied as a contrast-enhancing technique. Whereas spectral characteristics like color or thermal emission depend on the materials that make up the objects in a scene, the polarization properties depend strongly on the surface shape, orientation and roughness. The result is that spectral and polarization information often provide independent features for detection of an object that has similar spectral characteristics as the background or is hidden in a cluttered environment. The cause for this is that either polarization is created by reflection of (sun)light upon a dielectric or metallic surface or by refraction of thermal emission at such a surface, or that light is depolarized by the target in a different way than its surrounding objects. Imaging polarimeters for target detection need to be fast and deliver real-time data. The polarimetric configurations are therefore often as simple as possible, involving beam-splitting systems ("division of amplitude" or "division of aperture" ${ }^{1}$ ) or micropolarizer arrays on 
top of the focal-plane array ("division of focal plane"). See section 4 for an in-depth discussion of polarizationmeasurement techniques. Fig. 2a contains an example of a system with two synchronized thermal IR cameras behind a regular polarizing beam-splitter that can therefore provide the first two Stokes parameters in real time. Fig. $2 \mathrm{~b}$ presents a result from a micropolarizer system, which also provides real-time results.

\section{Intensity}
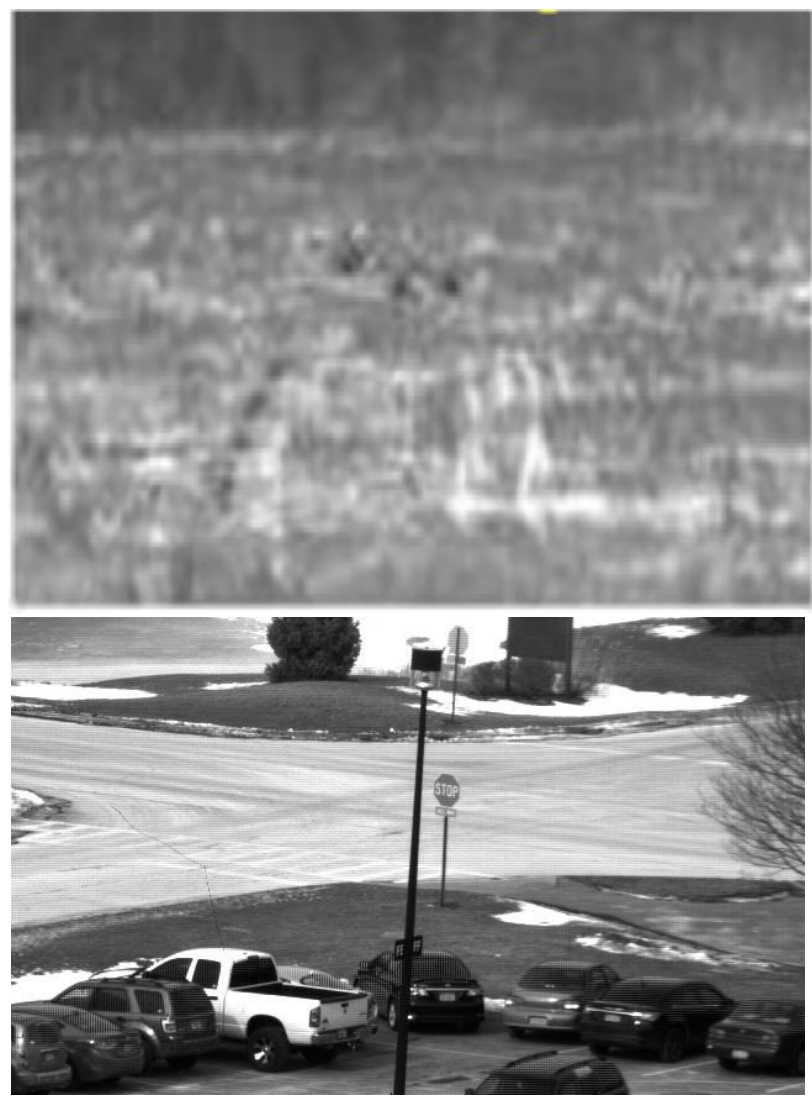

\section{Polarization}
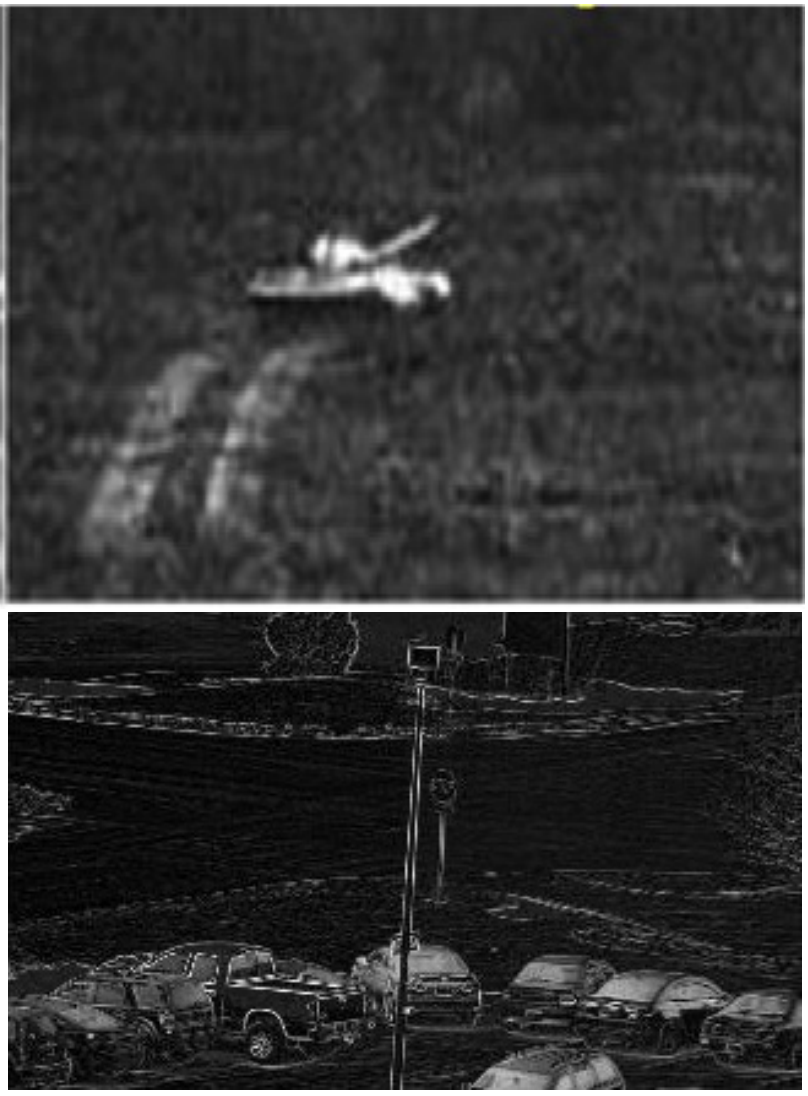

Figure 2. a) Tank in thermal equilibrium, imaged with an infrared camera in intensity and degree of linear polarization (courtesy: David Cenault, Polaris). b) Cars imaged with a microgrid polarizer focal-plane array. In degree of linear polarization only the windscreens stand out (courtesy: Dmitry Vorobiev).

\subsection{Astronomy}

In astronomy, the goal for polarimetric instrumentation ${ }^{2,3,15,16}$ is often required to detect tiny polarization signals $\left(\sim 1 \%\right.$ down to $\left.10^{-6}\right)$. We distinguish imaging polarimetry that is often used to reveal circumstellar structures (see Fig. 3a) and is being employed to directly image exoplanets, and spectropolarimetry that can probe the physical environment of stars and other astronomical objects through line polarization. For instance, a magnetic field splits up spectral lines according to the Zeeman effect. The split line components are all differently polarized, ${ }^{2}$ and hence sensitive polarimetry can probe $3 \mathrm{D}$ magnetic structure, even if the magnetic field is so small that the spectral line splitting is not measurable in intensity. The largest polarization effect is in circular polarization for a magnetic field component along the line-of-sight, and this effect has been used for more than a hundred years now (since G. E. Hale in 1908) and is now routinely employed at ground-based and satellite solar telescopes to monitor solar activity (see Fig. 3b). The same techniques are now used to measure and map (!) magnetic fields on unresolved stars, by employing also the Doppler effect and rotation-phase-resolved highly sensitive spectropolarimetry. In general, the light from astronomical targets is polarized whenever some departure from spherical symmetry is present, which is even the case for stars. Therefore, polarimetry enables 


\section{Intensity}

\section{Polarization}
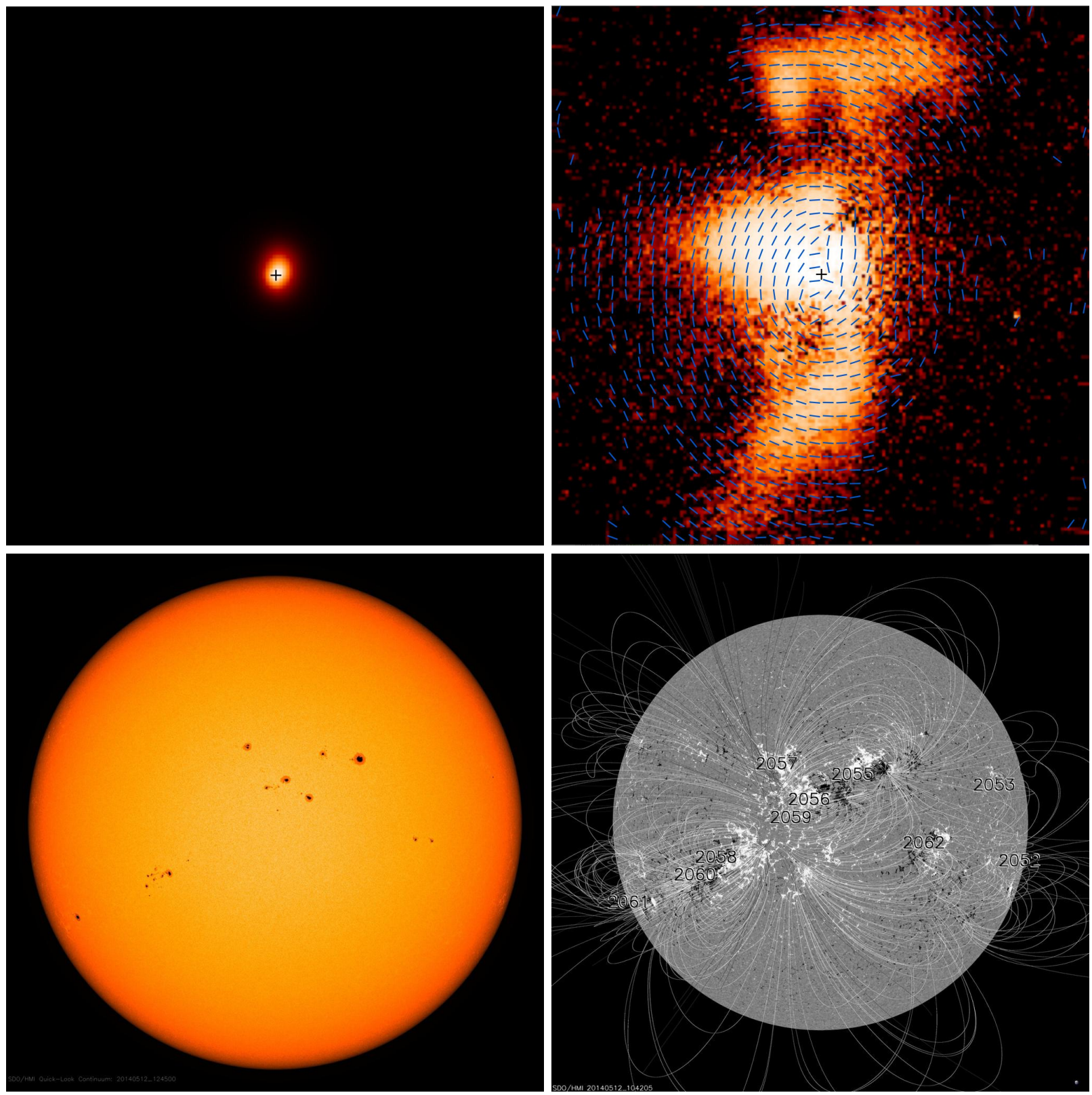

Figure 3. a) $\operatorname{ExPo}^{17}$ observations of the young star $\mathrm{T}$ Tauri. Sensitive imaging polarimetry reveals the structure of the circumstellar disk, as its material scatters the light from the central star (courtesy: Michiel Rodenhuis). b) Solar full-disk images from the SDO-HMI satellite instrument. Polarimetric measurements in a Zeeman-sensitive spectral line reveal the complex and dynamic solar magnetic field structure (from http://sdo.gsfc.nasa.gov/data/).

probing of spatial structures that are way beyond the capabilities of adaptive optics at large telescopes and long-baseline interferometry.

To reach high polarimetric sensitivity, several systematic effects need to be overcome. First and foremost, atmospheric turbulence creates "seeing" (i.e. twinkling of stars), which limits polarimetry if consecutive images are combined to form a polarization measurements. Simultaneous measurements after a polarizing beam-splitter suffer from differential effects that limit the polarimetric sensitivity to $\sim 10^{-3}$. Therefore, many astronomical 
polarimeters combine polarization measurement techniques to cancel out systematic effects (the so-called "dualbeam approach", see subsection 4.3). Another solution is to implement temporal polarization modulation that is faster than the seeing $(\sim 1 \mathrm{kHz})$, and measure the polarization information at the focal plane with a single set of pixels. As large-format, low-noise imaging detectors can generally not be read out at $\mathrm{kHz}$ rates, special demodulating detectors have been developed ${ }^{18,19}$ that combine a CCD with a stripe-mask and charge-shuffling that is synchronized with the fast polarization modulation. The polarization measurement is then built up in two (or more) interlaced detector images, that are read out after many thousands of polarization modulation cycles. Through such methods (or combinations thereof), polarimetric sensitivities are achieved that are only limited by photon noise. In that case, only the collection of more photons can improve upon the polarimetric sensitivity. Hence, large telescopes are necessary to collect these photons (even for solar observations!), but the photon noise can also be pushed down by binning multiple observations, binning pixels, or combining many spectral lines with similar polarization signals. ${ }^{20}$

Modern telescopes generally also limit the polarimetric performance, as polarimetric instrumentation is often located behind several telescope mirrors, that both induce polarization that is often larger than the target polarization, and modify incoming polarization ("cross-talk"). These instrumental polarization effects are frequently variable as they change with telescope pointing, ${ }^{21}$ and with aging and pollution of the mirrors. To fulfill demanding requirements upon the polarimetric accuracy, elaborate polarization strategies have to be devised (see subsection 4.5).

One of the most exciting challenges for astronomical polarimetry is to furnish direct observations of rocky exoplanets in the habitable zone. The contrast between such planets and their host stars is $\sim 10^{-7}-10^{-10}$, and, as the reflected light off the planet is polarized whereas the starlight can be considered unpolarized, sensitive polarimetry can be used to bridge that contrast, when coupled with extreme adaptive optics at extremely large telescopes and advanced coronagraphic techniques. Once such planets are detected in polarized light, the polarization signal as a function of wavelength and as a function of the planetary phase angle (i.e. the orbital motion of the planet) can yield signatures of habitability like liquid water oceans and clouds, and biosignatures as atmospheric oxygen. For such exoplanet characterization, also high polarimetric accuracy is required. Also the ultimate biosignature could be provided by polarimetry, as all complex molecules that are involved in biological processes (like amino acids, sugars, DNA and chlorophyll) are subject to homochirality: evolutionary processes have made that only one handedness in the chemical structure prevails. This symmetry breaking leaves an imprint upon circular polarization, ${ }^{22}$ although these signatures are yet at least an order of magnitude smaller than the linear polarization signatures from a planetary atmosphere and surface. The exciting science goal of finding extraterrestrial life therefore poses tremendous challenges to future polarimetric instrumentation.

\subsection{Biomedical Diagnostics}

Polarimetric instrumentation for biomedical diagnostics ${ }^{23-25}$ has additional design freedom, as also the polarization of the light source can be manipulated. In some cases, the light source is linearly polarized, and the scattered light from, e.g., tissue is analyzed in perpendicular and crossed polarization. ${ }^{25}$ Superficial, single-scattered light emerges mostly with the same polarization as the input, whereas deep multiple-scattered light also has a perpendicularly polarized component. The purely superficial tissue structure is the easily obtained from a polarimetric difference measurement. The degree of polarization associated with this measurement is a diagnostic for tissue properties, and can be used for early cancer detection, see Fig. 4a.

Such a set-up is generalized by implementing a Polarization State Generator (PSG) in the input beam that can generate several or all (fully polarized) Stokes parameters. The polarimeter in the output beam is then called a Polarization State Analyzer (PSA), and the overall instrument can be used to measure (part of) the Mueller matrix of tissue or a biological sample. This Mueller matrix can the be interpreted in terms of depolarization, retardance (amount and orientation) and polarizance. ${ }^{28}$ In turn, these parameters can be interpreted in terms of tissue properties. Depolarization can be caused by multiple scattering at isotropic structure, or by multidomain birefringent structures. Oriented fiber structures (e.g. collagen and neural axons) can generally be modeled as oriented retarders (and/or partial polarizers). An example of such Mueller matrix diagnostics is presented in Fig. 4b, for which the differences in depolarization indicate cancerous tissue. 

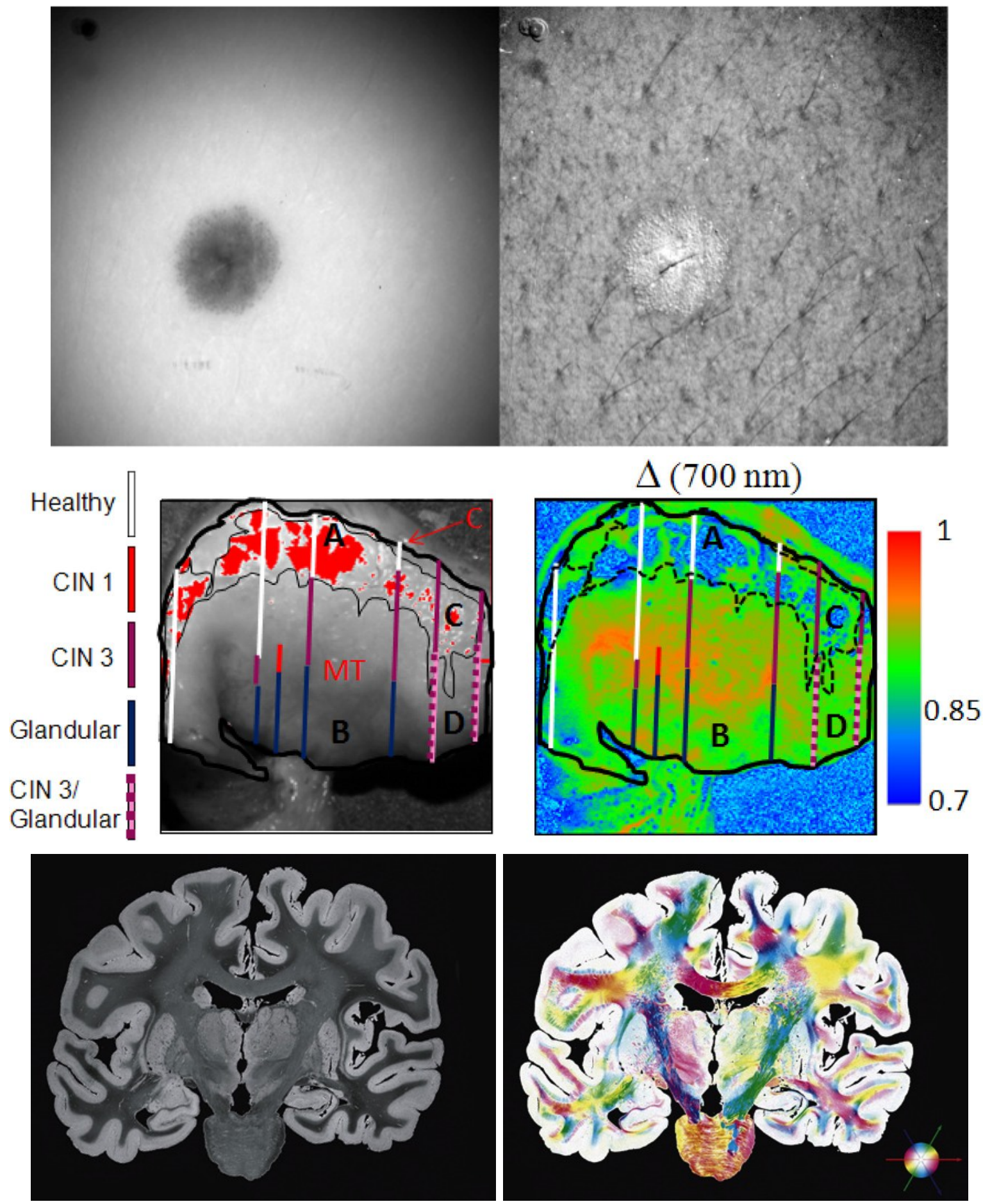

Figure 4. a) A mole (compound nevus) imaged through a polarizer parallel to the light source polarization, and in degree of linear polarization, which enables diagnostics of the risk of melanoma (courtesy: Steven Jacques). b) Intensity image and depolarized derived form Mueller matrix polarimetry of cancerous uterine cervix. The cuts indicate the results from pathology, which are instantaneously confirmed by the polarimetric imaging (adapted from Ref ${ }^{26}$ ). c) 3D mapping of fiber tracts in the brain enabled by polarization microscopy of brain slices: intensity image and reconstructed fiber geometry (adapted from $\operatorname{Ref}^{27}$ ). 
For similar measurements of internal organs, the input and output needs to occur through optical fibers. ${ }^{23}$ Generally, high-quality fibers do not depolarize or induce polarization, but they do act as retarders, and their retardance properties change with the movement of the fiber. If the PSG and/or PSA are located on the external end of the endoscope, these effects need to be monitored, calibrated and/or compensated for in quasi-real time. ${ }^{29}$ In the case of a depolarization measurement, these effects can be considered inconsequential by measuring the breaking of orthogonality of polarization states. ${ }^{30}$ It remains a technical challenge to design a polarimetric implementation at the internal fiber end.

Another application of biomedical polarimetry is microscopy of samples. Beautiful movies of cellular dynamics can be made by enhancing the contrast in a polarizing microsope, see www.openpolscope.org. Also slices of brain tissue can be fully characterized using a polarimetric approach. The retardance measurement allows for a 3D mapping of neural fiber structure, see Fig. 4c.

\subsection{Other Applications}

Polarimetry can be used to one's advantage in many different areas of application. Here we provide a nonexhaustive list:

- ophtalmology, glaucoma detection

- surveillance

- crop monitoring

- dehazing

- ocean monitoring

- underwater vision

- landmine detection

- erosion detection

- food quality control

- glass bottle production

- sugar measurements

- surface/material characterization, identification

- profilometry (3D shape retrieval)

- metrology
- cosmetics

- gas detection

- chiral molecules measurements

- corrosion detection

- measurement/imaging of birefringence: polymer sheets, windows, coatings, LC displays

- autonomous robotic vision (guidance)

- forensics, blood splash vector of impact

- road inspection

- ice detections airplanes

- measurement/characterization of nanoparticles

- ellipsometry: in-situ coating/layer characterization

- etc.

\subsection{Challenges, Commonalities \& Differences}

It should be clear from the previous subsections that polarimetry is still very much evolving to furnish a wide range of exciting applications. Many new developments are enabled by new technologies that are becoming available (see section 5). Some general trends can be observed for modern polarimetric instrumentation.

\subsubsection{Polarization vs. Depolarization}

In many applications of polarimetry such as astronomy and atmospheric monitoring, it may be impractical to actively illuminate the objects of interest. In these cases, the polarimeter is inherently a Stokes polarimeter that measures the polarization state of light leaving the scene. When the source of the radiation is unpolarized (e.g. the sun), then the objects of interest must polarize the light in order to have a polarization signature. In general, the degree of polarization to be measured is small, and the instrument needs to deal with a wide range of systematic effects and noise to be able to detect the relevant signals. The overall challenge is to design and 
build more sensitive polarimeters that will discover many more polarization signals, and open up new application space. When the source of the radiation is under the control of the observer, then the polarizing and depolarizing effects can all be probed. Whereas there are four degrees of freedom in the measured Stokes parameters, there are as many as 16 degrees of freedom when depolarization is considered. These additional DoFs make it possible to probe a much wider variety of scenes that are predominantly depolarizing in nature.

\subsubsection{From Contrast Enhancement to Characterization}

Polarimetry has long been regarded as a very valuable contrast-enhancing tool, not only for target detection, but also, e.g., for cloud detection in atmospheric research, skin cancer screening, and exoplanet detection in astronomy. However, in addition to object detection, polarized signals can also be used for object characterization. This generally means that polarization signals are to be measured as a function of (scattering) angle, and/or as a function of wavelength. The latter drives an important requirement to build polarimetric instrumentation that works over large spectral ranges, which, in turn, drives a demand for polarization optics (polarizers, retarders) that perform over large spectral ranges. This is not always trivial, particularly for wave plates, which are almost by definition chromatic. Most of all, polarimetric characterization also imposes (stringent) requirements upon the polarimetric accuracy of the instrument. Therefore, careful systems design and polarimetric calibration (see subsection 4.5) is becoming ever more important.

\subsubsection{Modularity with other Techniques}

Polarimetry is almost never a stand-alone technique. It needs to be optimally integrated in an optical system that also furnishes imaging, spectral, multi-angle and/or interferometric capabilities. ${ }^{31,32}$ Also, new optical techniques as lightfield imaging and orbital angular momentum manipulation could be paired with polarimetry. However, the optical system may not always be favorable in terms of polarization properties, and the polarization optics may degrade the performance of the other modalities of the instrument. Moreover, often the polarimeter is designed as an add-on, and is therefore not optimized at a system level. In such a case, the polarimeter is also very vulnerable to become a descope option. It is therefore a major challenge for the community to develop mature systems engineering approaches for polarimetry (subsection 4.5).

\section{POLARIZATION MEASUREMENT TECHNIQUES}

Polarimetry cf. the Stokes formalism entails the manipulation of light such that several independent intensity recordings can be combined to yield a (partial) measure of the polarization state of the incident light. The goal for every polarimeter design is to effectuate these intensity recordings in the most optimal way, taking into account the specific systematics associated to the application(s). Many implementations and several measurement domains are available for polarization measurements: the spatial domain, the temporal domain, the spectral domain, the angular domain, etc. In the target detection / remote sensing community this polarimetric process is categorized with "division of ..." labels. ${ }^{1}$ In the astronomical community, each implementation that enables a polarization measurement is called "modulation", e.g. spatial, temporal, spectral modulation. ${ }^{2}$ In both cases, this nomenclature is not applicable to all polarimeters. For instance, a polarimeter employing a polarizing beamsplitter is denominated with "division of amplitude" or named a manifestation of "spatial modulation", while it is not modulating in the strict sense of the definition. Another example would be that "spectral modulation" does not have an equivalent term "division of ...". Instead of lingering in semantic discussions, we provide an overview of polarization measurement techniques, and identify some new developments and opportunities.

\subsection{Measurement Domains}

A wide range of solutions exist to measure polarization using several detectors or different parts of a single detector, which we will generally designate to the spatial domain. The most straightforward implementation is the use of a polarizing beam-splitter, that could for instance deliver two beams, which difference provides a measure for the first and second Stokes parameter $\left(\left[S_{0}, S_{1}\right],[I, Q]\right)$. Compound beam-splitter assemblies can be designed that yield four beams to record the full linear polarization, or even full-Stokes data. ${ }^{33}$ Another implementation consists of boresighted optical systems, each with their own polarization analyzer and detector ("division of aperture"). The polarimetric system can also be implemented at the detector level, using microgrid 
polarizer arrays (see subsection 5.2) such that a combination of intensity measurements by clusters of pixels yields instantaneous polarization data ("division of focal plane"). Finally, various optical implementations ${ }^{32,34,35}$ have been developed to deliver a sine-curve modulation on top of the focal-plane image.

Other common polarimeter designs involve sequential measurements, i.e. "temporal modulation", or "division of time". Classical implementations include rotating polarizers and rotating retarders in combination with a fixed polarizer. Modern polarimeters often include faster temporal modulation enabled by liquid crystal components (see subsection 5.1): Liquid Crystal Variable Retarders (LCVRs) with fixed axes and electronically controllable retardance, or Ferroelectric Liquid Crystals (FLCs), which have a fixed retardance but a switchable axis orientation. A large advantage of such components is that they do not physically rotate. Other (very) fast temporal modulators include photo-elastic modulators (PEMs) and Pockels cells.

A relatively recent development is to deploy the spectral domain for the polarization measurement. ${ }^{32,35-39}$ A "channeled spectropolarimeter" intentionally creates an instrument matrix that is a periodic function of wave number by applying thick birefringent crystals that exhibit very chromatic retardances. This serves as a modulation that introduces side-bands in interferogram space that can be measured with either an interferometric or diffractive spectrometer. In general, several sinusoidal carriers of polarization information are present in the spectrum, although one can also optimize for just linear polarization. ${ }^{37}$

All these measurement domains for Stokes polarimetry can obviously also be generalized to Mueller matrix polarimetry. ${ }^{40,41}$

\subsection{Optimization}

Over the past 15 years, significant advances have been made independently in both the remote sensing/target detection community and the astronomy community in understanding the optimization of polarimeters in the presence of noise, measurement errors and other systematic effects. Since polarimeters are indirect sensing systems, the desired polarization parameters must be inferred from a set of direct measurements, and hence there is a system of equations that must be solved. As with all multi-dimensional sensing systems, the overall performance, SNR, and image dimensionality depend on a proper choice of the measurement basis. The simplest and most widely known method to optimize a polarimeter is to ensure that the "instrument matrix" or "modulation matrix" and its inverse (sometimes known as the "data reduction matrix" or "demodulation matrix") are 'well-conditioned' mathematically, or equivalently that the individual measurements made by the polarimeter are as independent as possible. The most optimal matrix inversion for an overdetermined system is the pseudoinverse. The various optimization metrics that have been established in the literature ${ }^{42-48}$ in the end all yield the same results. For instance, the four intensity measurements that are optimally converted into full-Stokes data are to be most widely spaced in the 3D space spanned by the polarized Stokes parameters, i.e. the Poincaré sphere. The solid body that is spanned by these four optimal polarization measurements is a tetrahedron, and this construction is widely used with many different implementations. ${ }^{43,49,50}$ In general, any modulation cf. an equilateral polygon or Platonic solid inside the Poincaré sphere is optimal with respect to noise propagation to the (fractional) Stokes parameters. For modulations that are more densely sampled in terms of sine/cosine curves, their orthogonality indicate to what extent the modulation is optimal. ${ }^{39}$ The single sine curve to measure degree and angle of linear polarization is of course well known for a rotating polarizer or a rotating half-wave plate system, but it can also be realized in the spectral domain ${ }^{37}$ or in the spatial domain. ${ }^{35,51}$ One could even argue that micropolarizer arrays constitute the critically sampled version of such spatial sinusoidal modulation. One astronomical instrument ${ }^{52}$ creates a spatial sinusoidal modulation in the form of a ring for point-source objects by rapidly rotating a polarizer and a wedge prism.

The concept of polarization modulation optimization is currently being applied to spectropolarimetry over very large wavelength ranges. Classically, one would design rotatable achromatic retarders by combining birefringent materials that compensate each other's retardance dispersion and/or by employing the Pancharatnam principle, which achieves achromatization by stacking (identical) retarders at different orientations. For a full-Stokes measurement it is often sufficient to demand that at every wavelength the (temporal) polarization modulation is optimal. ${ }^{53,54}$ This means that both the modulation process as well as the demodulation can be very chromatic. With this so-called "polychromatic modulation" one optimally uses all degrees of freedom in the design to reach as wide a spectral range as possible and/or optimize the polarimetric performance. One can also balance the 
noise propagation over the various Stokes parameters. And one can also further optimize the design space to minimize the effects of temperature, incidence angle and polarized fringes upon the temporal polarization modulation by a the optimized retarder stack. ${ }^{55,56}$ This "polychromatic" approach is also particularly useful to design a modulator package consisting of rapidly switching FLCs in combination with fixed retarders to furnish a large spectral range. ${ }^{57}$

For Mueller matrix polarimetry, usually not all Mueller matrix elements need to be determined with high accuracy. Similar optimization approaches can therefore be implemented to minimize the noise propagation for the essential Mueller matrix elements. ${ }^{58,59}$

\subsection{Dual-beam Polarimetry}

In many cases, the classical polarimetric implementations are limited in polarimetric sensitivity by systematic effects. For instance, the sequential measurements for temporal modulation also record inherent or apparent changes of the scene. For any kind of remote sensing smearing is caused by motion of the source (e.g. moving target or patient) and/or by motion of the observation platform (e.g. the satellite). In the case of astronomy polarimetric artifacts are created by the very rapid atmospheric turbulence that makes the stars twinkle. Also instantaneous polarization measurements after a polarizing beam-splitter are subject to differential effects: even after calibration, some differences in the transmissions of the two beams remain, and the gain table calibration of the detector(s) is limited to the $\sim 10^{-3}$ level. Moreover, the two beams will generally suffer from slightly different optical aberrations. All these effects induce to spurious polarization signatures.

By combining the temporal and spatial polarization measurement approaches, one can cancel out systematic effects (to first order). In the simplest implementation of this technique ("beam exchange" or "spatio-temporal modulation") the polarimeter consists of a rotatable half-wave plate (for linear polarimetry) or quarter-wave plate (for circular polarimetry) and a polarizing beam-splitter. For the case of circular polarimetry, two simultaneous recordings are made for one orientation of the quarter-wave plate, that yield measures of left- and right-handed circular polarization, respectively. After rotating the quarter-wave plate by $90^{\circ}$, the recordings have swapped: right- and left-handed circular polarization are now measured. These four recordings carry sufficient redundancy to demodulate to fractional circular polarization, regardless of the systematic effects in time and between the two beams. The data reduction can be carried out using either a double difference or a double ratio method. ${ }^{2,16,60,61}$ The measurement of fractional linear polarization is equivalent. This beam-exchange method is widely used in astronomy to reach polarimetric sensitivities down to $10^{-5}$.

This dual-beam approach can be generalized for more intricate temporal modulation schemes. In this case, both beams are first demodulated separately, and the results are then combined to cancel out the effects due to systematic temporal effects. ${ }^{62}$

\subsection{Multidomain Modulation}

Having established many polarization measurement methods in the spatial, temporal and spectral domains, and some combinations thereof, it is interesting to generalize these approaches and identify the potential for more hybridization. Fig. 5 presents a Venn diagram that categorizes polarization measurement/modulation approaches. The intersections of the domains are obviously the areas of interest.

The previous subsection already introduced the "beam exchange" and "dual-beam" methods that are used to suppress systematic effects. In addition to systems with a polarizing beam-splitter, probably also other spatialdomain polarimeters can benefit from additional information delivered by temporal modulation. In particular, artifacts that are present in polarization data from microgrid polarimeters (see Fig.2b) could be suppressed by such a hybrid approach. ${ }^{63}$

A similar approach is being used to suppress aliasing effects in channeled polarimetry. In general, the spectral/spatial polarization modulations cannot be distinguished from true spectral/spatial structure. By implementing a polarizing beam-splitter, one records two complementary sets of data, which sum represents the original, unmodulated spectrum or scene. The normalized difference then yields the unaliased polarization modulation. ${ }^{37}$ The same dealiasing can be accomplished with temporal modulation to shift the modulation pattern by $180^{\circ} .64$

Proc. of SPIE Vol. 9099 90990B-12 


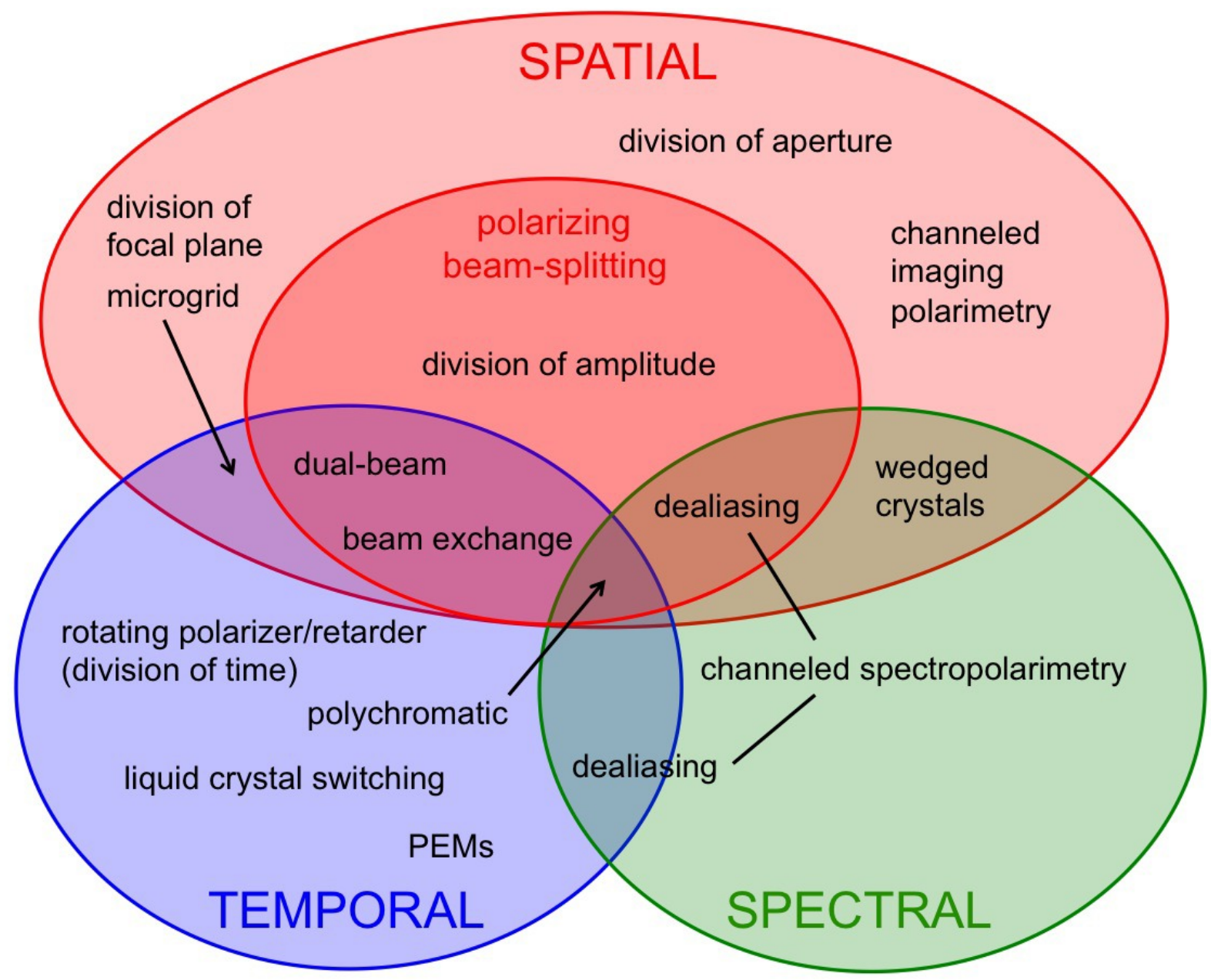

Figure 5. Venn diagram of polarization measurement/modulation domains.

The center of the Venn diagram is not taken yet, but it could be by the dual-beam polychromatic modulator. As this modulation is generally very chromatic, each modulation state (i.e. each orientation of the optimized retarder) yields sinusoidal modulations for all the Stokes parameters as a function of wavelength. ${ }^{55,56}$ In other words, this modulator can also be a spectral modulator, and it could be optimized for its spectral modulation orthogonalities $^{39}$ as well.

By considering all possible polarization modulation domains, all possible bandwidths ${ }^{65,66}$ of an instrument can be fully used. Moreover, one can then also allocate bandwidth to deal with systematic effects and aliasing, as in the case of the dual-beam approaches for temporal and spectral modulation. Furthermore, bandwidth can also be used to diagnose systematic effects. For instance, by rotating a quarter-wave plate to four positions separated by $90^{\circ}$, one can not only cancel out the effects due to offsets of the quarter-wave plate's retardance and orientation, but also demodulate to the "null profiles" that are diagnostics for the polarimetric noise level and the presence of systematic effects. ${ }^{60}$

\subsection{Systems Engineering \& Calibration}

Whereas systems engineering tools and error budget approaches are well-established for most areas of optics, this is not the case for polarimetric instrumentation. This is mostly because polarization errors are matrixquantities ${ }^{67}$ and mostly systematic in nature. Moreover, there is a very wide and diverse range of error sources, and the propagation of non-ideal properties can combine into second-order effects. ${ }^{68}$ Nevertheless, such an 
engineering tool-kit would be highly desirable for the design process of polarimetric instrumentation, to ensure that their performance will meet the requirements on, e.g., polarimetric sensitivity and accuracy.

One crucial factor to a successful polarimeter is its polarization calibration, for which known polarization states or components are applied to measure the polarimetric response. The only polarization state that can be created with full confidence is $100 \%$ polarized light created by a high-quality polarizer. The creation of $0 \%$ polarized light is already much more cumbersome, as all light sources are polarized to some degree. However, calibration with truly unpolarized light is sometimes necessary to determine the zero-point of the polarization measurement scale. Satisfactory depolarization may be achieved using an integrating sphere, low-quality optical fibers, dedicated "depolarizers", or a combination thereof. However, each of these methods generally relies on averaging in some domain (spatial, temporal, angle, spectrum), and care must be taken to ensure that the instrument being calibrated does not resolve too finely in a domain that requires averaging. Calibration with known polarization states with low polarization degree may be required as many polarimeters are designed to measure small amounts of polarization, and they may exhibit non-linear effects upon $100 \%$ polarized input. Controlled linear polarization can be created from a depolarized light source by tiltable (and rotatable) glass plates with know refractive index and coating properties. But in the end, the question is always how well one can calibrate the calibration optics. Such an overall calibration can be obtained by fitting a model to both the calibratible optical train and the calibration optics themselves. The calibration unit could then consist of a rotatable polarizer and a rotatable quarter-wave plate, that together can create any $100 \%$ polarized Stokes vector. By creating an overdetermined set of calibration states, all parameters in the model can be fit in a least-squares sense, including rotation and retardance offsets of the calibration optics. ${ }^{2,69}$ For Mueller matrix polarimeters, elements with some known Mueller matrix properties can be inserted, and through an eigenvalue analysis both the PSG and PSA matrices and the calibration components can be determined. ${ }^{70}$

As the polarimetric accuracy relates the measured Stokes parameters to the true ones, it can never be fully validated by calibration. Stability of the data or the calibration results, or inter-instrument comparisions are often the best indication that the polarimeter is accurate. Standard targets (e.g. unpolarized/polarized stars), scenes (depolarizing surfaces, the polarized sky ${ }^{21}$ ) and spectral lines can be used to verify the calibration, or to indeed recalibrate the system. Also, one can employ symmetries in images (e.g. scattering polarization is always perpendicular/parallel to the scattering plane) or spectra (e.g. the Zeeman effect creates purely symmetric and antisymmetric profiles for spectral lines formed in a uniform medium) to check the calibration.

\section{MODERN POLARIZATION TECHNOLOGIES}

Many developments in polarimetric instrumentation are driven by technological developments, that are often not initially geared towards a polarimetric application. We list a few novel developments.

\subsection{Liquid Crystal Components}

Although Liquid Crystal Variable Retarders constitute probably the most common polarization modulator nowadays, they are still evolving. As they switch more slowly than their FLC cousins, considerable effort has been invested by companies such as Meadowlark to develop "swift" LCVRs with $\sim 1$ ms switching time. Moreover, technical solutions now exist to build wide-field and achromatic LCVRs. ${ }^{71}$ Active liquid crystal elements have also been space-qualified, ${ }^{72}$ and are finding their way into designs for satellite-based polarimeters.

The twisting nature of some nematic liquid crystal materials gives rise to a new method for achromatizing retarder optics. A generalization of the Pancharatnam principle can be applied by stacking (self-aligning) liquid crystal layers with controlled thickness, birefringence dispersion, and twist. With these so-called "multi-twist retarders" (MTR) unprecedented retardance performance over huge spectral ranges can be achieved. ${ }^{73}$

Half-wave retarders can also be used to manipulate the phase of a beam of light. In contrast to the classical phase which depends on physical optical path differences, such geometric or vector-phase can be applied by a perfectly flat optic, and operates on circular polarization states. Regardless of orientation, the half-wave retarder flips the handedness of the circular polarization, but the absolute phase of the emerging light linearly depends on the (local) orientation of the half-wave retarder, and the applied vector-phase is opposite for the opposite circular polarization states. A half-wave retardance structure with a continuously rotating fast axis in one dimension 

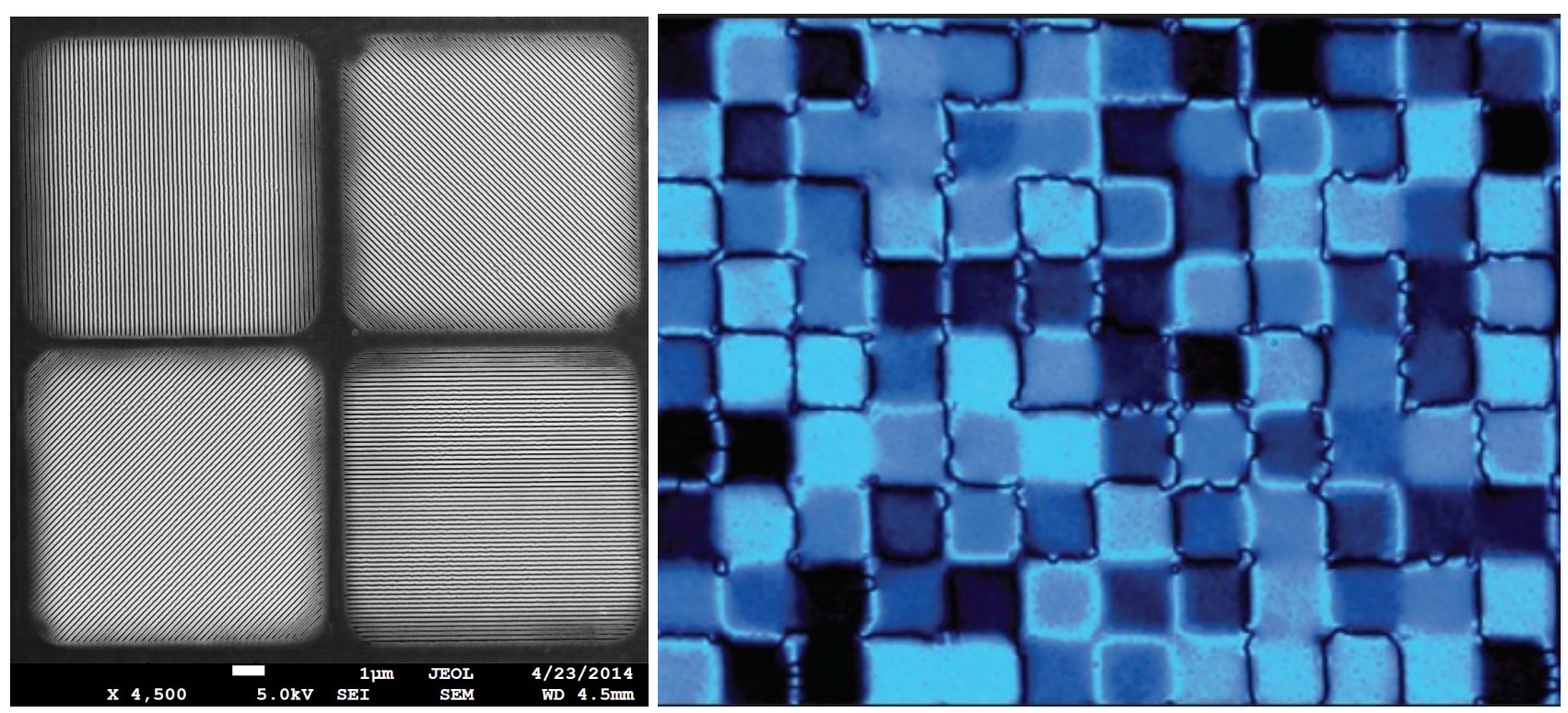

Figure 6. a) SEM image of four pixels of a micropolarizer array (courtesy: Neal Brock, 4D Technology; MoxTek). b) Microscopic image of a patterned retarder; each pixel is $10 \mu$ in size (courtesy: Michael Escuti).

therefore indeed functions as a "polarization grating" $:{ }^{74}$ left circular polarization is diffracted in order +1 , and right circular is diffracted in order -1 (or vice versa). With the addition of a quarter-wave plate, this device can simultaneously act as a regular polarizing beam-splitter and a dispersing element. Polarization gratings can be created using liquid crystal patterning, and achromatized using the MTR approach. With this development, essentially any phase pattern can be written ${ }^{75}$ (see Fig. 6b) and broadbanded. This opens up exciting avenues for joint phase and polarization control.

\subsection{Micropatterning}

Several technical solutions can now be employed to directly measure polarization at the pixel level. Microgrid polarizer arrays (Fig. 6a) can now be routinely fabricated using state-of-the-art lithographic techniques. Advances in lithography allow such write-grid structures to be written in ever smaller scales, such that their applicability moves down to UV wavelengths. An example of micropolarizer array data can be found in Fig. 2b, in which in the intensity image the modulating effect due to the polarizing windscreen is already apparent. The image in degree of polarization contains artifacts, which are the consequence of poor sampling of high spatial frequency image features by the focal plane. This could be mitigated by optimizing the sampling of the point-spread function by the imaging array. Also, dedicated Fourier-based reconstructor algorithms ${ }^{76,77}$ can be used to reduce aliasing. And indeed also the micropolarizer conguration could be modified to optimize the extraction of the polarization data. ${ }^{77}$

Micropolarizer arrays can only measure linear polarization. For full-Stokes applications a micropatterned retarder in combination with a regular polarizer has been developed. ${ }^{50}$

Note that the focal plane does not necessarily need to be sampled with pixels. Therefore also microlens arrays and (polarization-maintaining) fiber bundles ${ }^{78}$ can be used to sample the focal plane in combination with a polarimetric modulation implementation. ${ }^{31}$

\section{DATA INTERPRETATION AND VISUALIZATION}

\subsection{Data reduction \& Interpretation}

Polarimetric data reduction is often very much dependent on the application. Nevertheless, some techniques and approaches are of general interest. 
- Motion artifacts often limit polarimetric imaging. Such spurious polarization signals can be compressed by employing an optical flow technique to stabilize the raw images. ${ }^{79}$

- Artifacts can also be reduced by nonlocal mean filtering. ${ }^{80}$

- Image segmentation can be used to both optimize the SNR and the image reconstruction. ${ }^{81}$

- Mueller matrix data can be directly decomposed into physical components ${ }^{28}$ and physicality of the Mueller matrix can be imposed to suppress noise and measurement errors.

- In some cases it is better to directly model/interpret the raw modulated data, instead of demodulating to Stokes parameters

\subsection{Polarimetric Data Visualization}

Polarization data is by definition multidimensional, and therefore its visualization is challenging. We have included a few good examples of visualization of polarimetric data in this paper. Obviously vector maps are ideally suited for polarimetry, and many appealing visualization tools are available for that. Often, a HSB color scheme is employed to represent degree and angle of polarization, ${ }^{82}$ but it can even be used to visualize 3D data derived from polarization measurements (Fig. 4c). ${ }^{27}$ And as polarimetry is frequently not the only data modality, the multidimensional polarization is often to be fused with other diagnostics.

In the end, the Stokes parameters or Mueller matrix elements are almost never the end-product of a polarimetric instrument, and the visualization should be implemented in terms of the parameters that the user actually cares about. Dedicated application development is essential for the successful adoption of polarimetric instrumentation by non-specialist users. Polarimetric devices should be easy to use, and their output easy to understand. Miniaturization of polarimetric instrumentation enables everyday application by, e.g., a physician using a hand-held device, or a soldier receiving real-time input from a helmet-mounted device (Polaris product), or citizen scientists using a dedicated smartphone spectropolarimeter for atmospheric research. ${ }^{83}$

\section{CONCLUSIONS \& RECOMMENDATIONS}

\subsection{Exchange of Expertise}

Bringing together polarimetrists from a wide range of research fields proved very fruitful to exchange expertise and identify new opportunities for collaboration. Several multidisciplinary projects were established:

- Introduce the dual-beam technique developed for astronomical polarimetry to polarimetric instrumentation commonly used for remote sensing (e.g. micropolarizer arrays).

- Use high-accuracy blue-sky polarimetric measurements for aerosol research as input for telescope polarization calibrations.

- Introduce micropatterning technology and fiber bundles for in-vivo Mueller matrix polarimetry.

- Amalgamate polarization measurement techniques in to a generic multidomain modulation framework.

- Explore common calibration techniques for optical fibers and complex telescope optical paths.

- Apply Mueller matrix decompositions to scattering media (atmosphere, skin, circumstellar material, underwater environment).

- Introduce micropolarizer arrays to astronomy.

- Generalize "polychromatic" modulation with spectral modulation optimization.

- Etc. 


\subsection{Exchange of Information}

We conclude that it is clearly beneficial for all these different communities that apply polarimetry to exchange information in a regular basis. To enable that, we take the initiative for the following platforms:

1. We started the LinkedIn group "Optical polarimetry" for discussions about anything from recent publications to specialized questions.

2. We will create a dedicated wiki-style website that offers up-to-date information about optical polarimetry in general, and its range of applications in particular. This website will include recent review papers, a glossary of terms, definitions and conventions, good educational/outreach material, a gallery of polarization images, a list of applications and vendors, etc.

3. We aim to organize such an interactive, international, multidisciplinary workshop about optical polarimetry on a bi-annual basis. So we look forward to the next one in 2016.

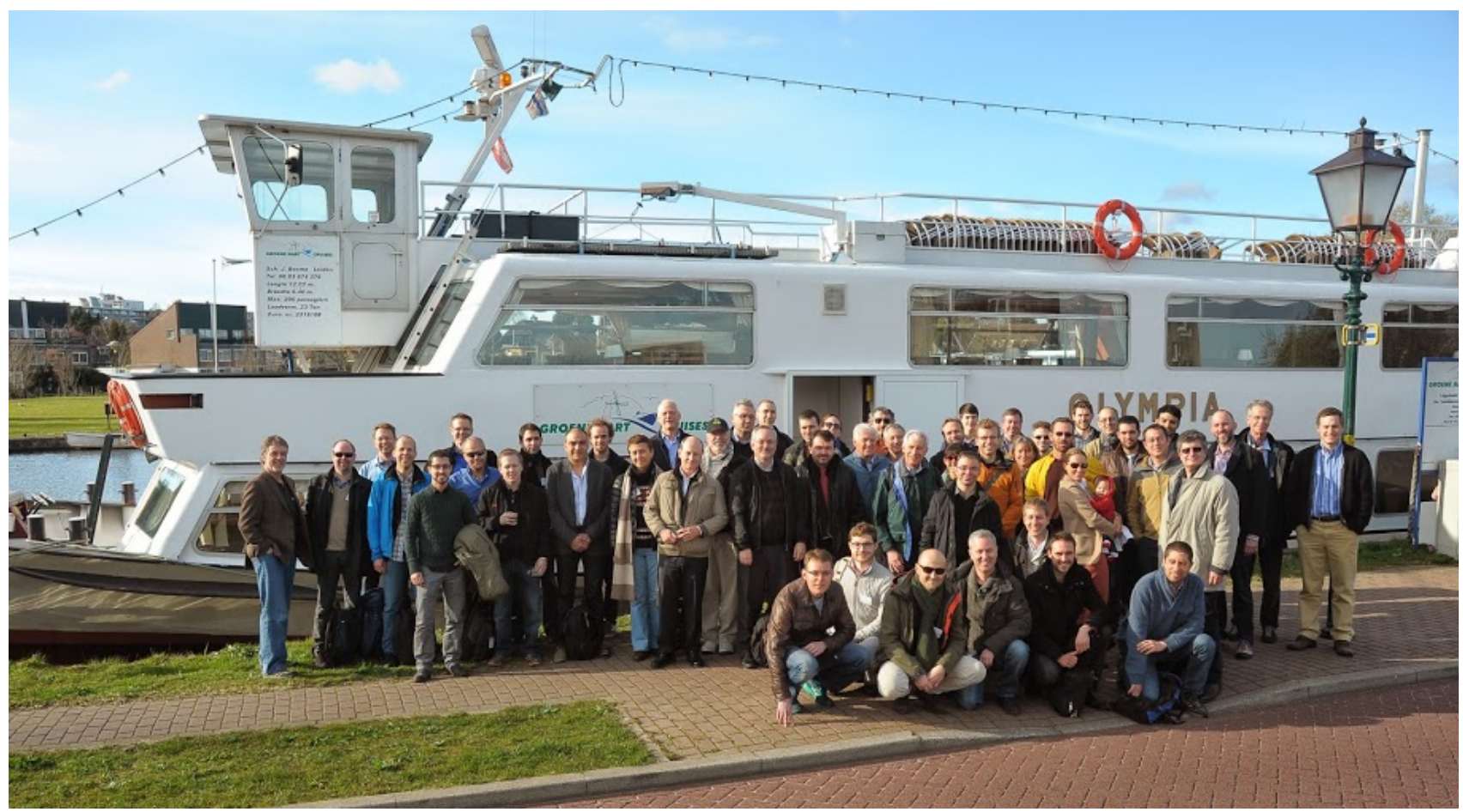

Figure 7. Most of the participants to the Lorentz Center workshop "Polarimetric Techniques \& Technology", held March 24-28, 2014 in Leiden, the Netherlands. Photo taken by the captain of the boat, using Joe Shaw's camera.

\section{ACKNOWLEDGMENTS}

The authors thank all the participants for their invaluable contributions to the workshop and to this publication. We thank the Lorentz Center and its staff for providing the ideal platform for our workshop, and for funding. We we thank the COST network MP1104 "Polarisation as a tool to study the solar system and beyond" for funding and for the initial ideas for organizing this multidisciplinary workshop. 


\section{REFERENCES}

[1] J. S. Tyo, D. L. Goldstein, D. B. Chenault, and J. A. Shaw, "Review of passive imaging polarimetry for remote sensing applications," Appl. Opt. 45, pp. 5453-5469, Aug 2006.

[2] F. Snik and C. U. Keller, "Astronomical Polarimetry: Polarized Views of Stars and Planets," in Planets, Stars and Stellar Systems. Volume 2: Astronomical Techniques, Software and Data, T. D. Oswalt and H. E. Bond, eds., p. $175,2013$.

[3] D. Clarke, Stellar Polarimetry, 2010.

[4] F. Snik, A. Lopez Artiste, and S. Bagnulo 2014.

[5] E. Wolf, Introduction to the Theory of Coherence and Polarization of Light, Cambridge University Press, 2007.

[6] I. J. Hodgkinson and Q. H. Wu, Birefringent Thin Films and Polarizing Elements, World Scientific, 1998.

[7] I. J. Vaughn and B. G. Hoover, "Noise reduction in a laser polarimeter based on discrete waveplate rotations," Opt. Express 16, pp. 2091-2108, Feb 2008.

[8] D. J. Diner, M. J. Garay, O. V. Kalashnikova, B. E. Rheingans, S. Geier, M. A. Bull, V. M. Jovanovic, F. Xu, C. J. Bruegge, A. Davis, K. Crabtree, and R. A. Chipman, "Airborne multiangle spectropolarimetric imager (airmspi) observations over california during nasa's polarimeter definition experiment (podex)," 2013.

[9] N. J. Pust and J. A. Shaw, "Dual-field imaging polarimeter using liquid crystal variable retarders," Appl. Opt. 45, pp. 5470-5478, Aug 2006.

[10] P.-Y. Deschamps, F.-M. Breon, M. Leroy, A. Podaire, A. Bricaud, J.-C. Buriez, and G. Seze, "The polder mission: instrument characteristics and scientific objectives," Geoscience and Remote Sensing, IEEE Transactions on 32, pp. 598-615, May 1994.

[11] B. Cairns, E. E. Russell, J. D. LaVeigne, and P. M. W. Tennant, "Research scanning polarimeter and airborne usage for remote sensing of aerosols," in Polarization Science and Remote Sensing, J. A. Shaw and J. S. Tyo, eds., Society of Photo-Optical Instrumentation Engineers (SPIE) Conference Series 5158, pp. 33-44, Dec. 2003.

[12] R. J. Peralta, C. Nardell, B. Cairns, E. E. Russell, L. D. Travis, M. I. Mishchenko, B. A. Fafaul, and R. J. Hooker, "Aerosol polarimetry sensor for the Glory Mission," in Society of Photo-Optical Instrumentation Engineers (SPIE) Conference Series, Society of PhotoOptical Instrumentation Engineers (SPIE) Conference Series 6786, Nov. 2007.

[13] D. J. Diner, A. Davis, B. Hancock, S. Geier, B. Rheingans, V. Jovanovic, M. Bull, D. M. Rider, R. A. Chipman, A.-B. Mahler, and S. C. McClain, "First results from a dual photoelastic-modulator-based polarimetric camera," Appl. Opt. 49, pp. $2929-2946$, May 2010.

[14] G. van Harten, F. Snik, J. H. H. Rietjens, J. M. Smit, J. de Boer, R. Diamantopoulou, O. P. Hasekamp, D. M. Stam, C. U. Keller, E. C. Laan, A. L. Verlaan, W. A. Vliegenthart, R. Ter Horst, R. Navarro, K. Wielinga, S. Hannemann, S. G. Moon, and R. Voors, "Prototyping for the Spectropolarimeter for Planetary EXploration (SPEX): calibration and sky measurements," in Society of Photo-Optical Instrumentation Engineers (SPIE) Conference Series, Society of Photo-Optical Instrumentation Engineers (SPIE) Conference Series 8160, Sept. 2011.

[15] C. U. Keller, "Instrumentation for astrophysical spectropolarimetry," in Astrophysical Spectropolarimetry, J. Trujillo-Bueno, F. Moreno-Insertis, and F. Sánchez, eds., pp. 303-354, 2002.

[16] J. Tinbergen, Astronomical Polarimetry, Sept. 1996.

[17] M. Rodenhuis, H. Canovas, S. V. Jeffers, M. de Juan Ovelar, M. Min, L. Homs, and C. U. Keller, "The extreme polarimeter: design, performance, first results and upgrades," in Society of Photo-Optical Instrumentation Engineers (SPIE) Conference Series, Society of Photo-Optical Instrumentation Engineers (SPIE) Conference Series 8446, Sept. 2012.

[18] H.-M. Schmid, M. Downing, R. Roelfsema, A. Bazzon, D. Gisler, J. Pragt, C. Cumani, B. Salasnich, A. Pavlov, A. Baruffolo, J.-L. Beuzit, A. Costille, S. Deiries, K. Dohlen, C. Dominik, E. Elswijk, M. Feldt, M. Kasper, D. Mouillet, C. Thalmann, and F. Wildi, "Tests of the demodulating CCDs for the SPHERE / ZIMPOL imaging polarimeter," in Society of Photo-Optical Instrumentation Engineers (SPIE) Conference Series, Society of Photo-Optical Instrumentation Engineers (SPIE) Conference Series 8446, Sept. 2012.

[19] D. M. Harrington and J. R. Kuhn, "Precision and Resolution in Stellar Spectropolarimetry," in Solar Polarization 6, J. R. Kuhn, D. M. Harrington, H. Lin, S. V. Berdyugina, J. Trujillo-Bueno, S. L. Keil, and T. Rimmele, eds., Astronomical Society of the Pacific Conference Series 437, p. 257, Apr. 2011.

[20] O. Kochukhov, V. Makaganiuk, and N. Piskunov, "Least-squares deconvolution of the stellar intensity and polarization spectra," $A \mathscr{E} A$ 524, p. A5, Dec. 2010.

[21] D. M. Harrington, J. R. Kuhn, and S. Hall, "Deriving Telescope Mueller Matrices Using Daytime Sky Polarization Observations," PASP 123, pp. 799-811, July 2011.

[22] W. B. Sparks, J. H. Hough, L. Kolokolova, T. A. Germer, F. Chen, S. DasSarma, P. DasSarma, F. T. Robb, N. Manset, I. N. Reid, F. D. Macchetto, and W. Martin, "Circular polarization in scattered light as a possible biomarker," JQSRT 110, pp. 1771-1779, Sept. 2009.

[23] T. Novikova, A. Pierangelo, A. D. Martino, A. Benali, and P. Validire, "Polarimetric imaging for cancer diagnosis and staging," Opt. Photon. News 23, pp. 26-33, Oct 2012.

[24] N. Ghosh and I. A. Vitkin, "Tissue polarimetry: concepts, challenges, applications, and outlook," Journal of Biomedical Optics 16(11), pp. 110801-110801-29, 2011

[25] S. L. Jacques, "Optical properties of biological tissues: a review," Physics in Medicine and Biology 58(11), p. R37, 2013.

[26] A. Pierangelo, A. Nazac, A. Benali, P. Validire, H. Cohen, T. Novikova, B. H. Ibrahim, S. Manhas, C. Fallet, M.-R. Antonelli, and A.-D. Martino, "Polarimetric imaging of uterine cervix: a case study," Opt. Express 21, pp. 14120-14130, Jun 2013.

[27] M. Axer, K. Amunts, D. Grssel, C. Palm, J. Dammers, H. Axer, U. Pietrzyk, and K. Zilles, "A novel approach to the human connectome: Ultra-high resolution mapping of fiber tracts in the brain," NeuroImage 54(2), pp. 1091 - 1101, 2011.

[28] J. J. Gil, "Review on mueller matrix algebra for the analysis of polarimetric measurements," Journal of Applied Remote Sensing 8(1), p. 081599, 2014.

[29] J. Desroches, D. Pagnoux, F. Louradour, and A. Barthélémy, "Fiber-optic device for endoscopic polarization imaging," Opt. Lett. 34, pp. 3409-3411, Nov 2009

[30] J. Fade and M. Alouini, "Depolarization remote sensing by orthogonality breaking," Phys. Rev. Lett. 109, p. 043901, Jul 2012.

[31] M. Rodenhuis, F. Snik, G. van Harten, J. Hoeijmakers, R. Joseph, and C. U. Keller, "Five-dimensional optical instrumentation: combining polarimetry with time-resolved integral-field spectroscopy," in Society of Photo-Optical Instrumentation Engineers (SPIE) Conference Series, Society of Photo-Optical Instrumentation Engineers (SPIE) Conference Series $9099,2014$.

[32] M. W. Kudenov, M. J. Escuti, E. L. Dereniak, and K. Oka, "White-light channeled imaging polarimeter using broadband polarization gratings," Appl. Opt. 50, pp. 2283-2293, May 2011. 
[33] E. Compain and B. Drevillon, "Broadband division-of-amplitude polarimeter based on uncoated prisms," Appl. Opt. 37, pp. 5938-5944, Sep 1998.

[34] K. Oka and T. Kaneko, "Compact complete imaging polarimeter using birefringent wedge prisms," Opt. Express 11, pp. 1510-1519, Jun 2003.

[35] W. Sparks, T. A. Germer, J. W. MacKenty, and F. Snik, "Compact and robust method for full stokes spectropolarimetry," Appl. Opt. 51, pp. 5495-5511, Aug 2012.

[36] K. Oka and T. Kato, "Spectroscopic polarimetry with a channeled spectrum," Opt. Lett. 24, pp. 1475-1477, Nov 1999.

[37] F. Snik, T. Karalidi, and C. U. Keller, "Spectral modulation for full linear polarimetry," Appl. Opt. 48, pp. 1337-1346, Mar 2009.

[38] K. H. Nordsieck, "A Simple Polarimetric System for the Lick Observatory Image-Tube Scanner," PASP 86, p. 324, June 1974.

[39] A. S. Alenin and J. S. Tyo, "Generalized channeled polarimetry," J. Opt. Soc. Am. A 31, pp. 1013-1022, May 2014.

[40] G. Anna, H. Sauer, F. Goudail, and D. Dolfi, "Fully tunable active polarization imager for contrast enhancement and partial polarimetry," Appl. Opt. 51, pp. 5302-5309, Jul 2012.

[41] N. Hagen, K. Oka, and E. L. Dereniak, "Snapshot mueller matrix spectropolarimeter," Opt. Lett. 32, pp. $2100-2102$, Aug 2007.

[42] J. S. Tyo, "Design of optimal polarimeters: Maximization of signal-to-noise ratio and minimization of systematic error," Appl. Opt. 41, pp. 619-630, Feb 2002.

[43] D. S. Sabatke, M. R. Descour, E. L. Dereniak, W. C. Sweatt, S. A. Kemme, and G. S. Phipps, "Optimization of retardance for a complete stokes polarimeter," Opt. Lett. 25, pp. 802-804, Jun 2000.

[44] J. C. del Toro Iniesta and M. Collados, "Optimum Modulation and Demodulation Matrices for Solar Polarimetry," Appl. Opt. 39, pp. 1637-1642, 2000.

[45] D. M. Harrington, J. R. Kuhn, C. Sennhauser, E. J. Messersmith, and R. J. Thornton, "Achromatizing a Liquid-Crystal Spectropolarimeter: Retardance vs. Stokes-Based Calibration of HiVIS," PASP 122, pp. 420-438, Apr. 2010.

[46] F. Goudail, "Noise minimization and equalization for stokes polarimeters in the presence of signal-dependent poisson shot noise," Opt. Lett. 34, pp. 647-649, Mar 2009.

[47] A. D. Martino, Y.-K. Kim, E. Garcia-Caurel, B. Laude, and B. Drévillon, "Optimized mueller polarimeter with liquid crystals," Opt. Lett. 28, pp. 616-618, Apr 2003.

[48] J. Zallat, S. Aïnouz, and M. P. Stoll, "Optimal configurations for imaging polarimeters: impact of image noise and systematic errors," Journal of Optics A: Pure and Applied Optics 8(9), p. 807, 2006.

[49] A. Peinado, A. Lizana, J. Vidal, C. Iemmi, and J. Campos, "Optimization and performance criteria of a stokes polarimeter based on two variable retarders," Opt. Express 18, pp. 9815-9830, May 2010.

[50] W.-L. Hsu, G. Myhre, K. Balakrishnan, N. Brock, M. Ibn-Elhaj, and S. Pau, "Full-stokes imaging polarimeter using an array of elliptical polarizer," Opt. Express 22, pp. 3063-3074, Feb 2014.

[51] M. W. Kudenov, M. E. L. Jungwirth, E. L. Dereniak, and G. R. Gerhart, "White light sagnac interferometer for snapshot linear polarimetric imaging," Opt. Express 17, pp. 22520-22534, Dec 2009.

[52] D. Clarke and D. Neumayer, "Experiments with a novel CCD stellar polarimeter," A\&A 383, pp. 360-366, Jan. 2002.

[53] S. Tomczyk, R. Casini, A. G. de Wijn, and P. G. Nelson, "Wavelength-diverse polarization modulators for Stokes polarimetry," Appl. Opt. 49, pp. 3580-3586, 2010.

[54] P. A. Letnes, I. S. Nerbø, L. M. S. Aas, P. G. Ellingsen, and M. Kildermo, "Fast and optimal broad-band Stokes/Mueller polarimeter design by the use of a genetic algorithm," Optics Express 18, pp. 23095-23103, 2010.

[55] F. Snik, G. van Harten, R. Navarro, P. Groot, L. Kaper, and A. de Wijn, "Design of a full-Stokes polarimeter for VLT/X-shooter," in Society of Photo-Optical Instrumentation Engineers (SPIE) Conference Series, Society of Photo-Optical Instrumentation Engineers (SPIE) Conference Series 8446, Sept. 2012.

[56] G. Van Harten, F. Snik, C. U. Keller, and A. G. de Wijn, "Design Analysis of a Polychromatic Full-Stokes Polarization Modulator for the 300-2500 nm Wavelength Range." Appl. Opt. (to be submitted), 2014.

[57] J. Hou, A. G. de Wijn, and S. Tomczyk, "Design and measurement of the Stokes polarimeter for the COSMO K-coronagraph," ApJ $\mathbf{7 7 4}$, p. 85, Sept. 2013.

[58] J. Tyo, Z. Wang, S. Johnson, and B. Hoover, "Design and optimization of partial mueller matrix polarimeters," Appl. Opt. 49, pp. 2326-2333, Apr 2010.

[59] G. Anna, F. Goudail, P. Chavel, and D. Dolfi, "On the influence of noise statistics on polarimetric contrast optimization," Appl. Opt. 51, pp. 1178-1187, Mar 2012.

[60] S. Bagnulo, M. Landolfi, J. D. Landstreet, E. Landi Degl'Innocenti, L. Fossati, and M. Sterzik, "Stellar Spectropolarimetry with Retarder Waveplate and Beam Splitter Devices," PASP 121, pp. 993-1015, Sept. 2009.

[61] M. Semel, J. Donati, and D. E. Rees, "Zeeman-Doppler imaging of active stars. 3: Instrumental and technical considerations," $A \& A$ 278, pp. 231-237, Oct. 1993.

[62] R. Casini, A. G. de Wijn, and P. G. Judge, "Analysis of seeing-induced polarization cross-talk and modulation scheme performance," The Astrophysical Journal 757(1), p. 45, 2012.

[63] C. F. LaCasse, T. Ririe, R. A. Chipman, and J. S. Tyo, "Spatio-temporal modulated polarimetry," 2011.

[64] J. Craven and M. W. Kudenov, "False signature reduction in channeled spectropolarimetry," Optical Engineering 49(5), pp. 053602$053602-10,2010$.

[65] C. F. LaCasse, R. A. Chipman, and J. S. Tyo, "Band limited data reconstruction in modulated polarimeters," Opt. Express 19, pp. 14976-14989, Aug 2011.

[66] C. F. LaCasse, J. S. Tyo, and R. A. Chipman, "Role of the null space of the drm in the performance of modulated polarimeters," Opt. Lett. 37, pp. 1097-1099, Mar 2012.

[67] M. de Juan Ovelar, F. Snik, and C. U. Keller, "M\&m's: an error budget and performance simulator code for polarimetric systems," in Society of Photo-Optical Instrumentation Engineers (SPIE) Conference Series, Society of Photo-Optical Instrumentation Engineers (SPIE) Conference Series 8160, Sept. 2011.

[68] C. U. Keller, "Recent Progress in Imaging Polarimetry," Sol. Phys. 164, pp. 243-252, Mar. 1996.

[69] H. Socas-Navarro, D. Elmore, A. Asensio Ramos, and D. M. Harrington, "Characterization of telescope polarization properties across the visible and near-infrared spectrum. Case study: the Dunn Solar Telescope," A 6 A 531, p. A2, July 2011.

[70] E. Compain, S. Poirier, and B. Drevillon, "General and self-consistent method for the calibration of polarization modulators, polarimeters, and mueller-matrix ellipsometers," Appl. Opt. 38, pp. 3490-3502, Jun 1999. 
[71] G. Capobianco, S. Fineschi, G. Massone, E. Balboni, A. M. Malvezzi, G. Crescenzio, L. Zangrilli, P. Calcidese, E. Antonucci, and M. Patrini, "Electro-optical polarimeters for ground-based and space-based observations of the solar K-corona," in Society of Photo-Optical Instrumentation Engineers (SPIE) Conference Series, Society of Photo-Optical Instrumentation Engineers (SPIE) Conference Series 8450, Sept. 2012.

[72] N. Uribe-Patarroyo, A. Alvarez-Herrero, P. García Parejo, J. Vargas, R. L. Heredero, R. Restrepo, V. Martínez Pillet, J. C. Del Toro Iniesta, A. López, S. Fineschi, G. Capobianco, M. Georges, M. López, G. Boer, and I. Manolis, "Space-qualified liquid-crystal variable retarders for wide-field-of-view coronagraphs," in Society of Photo-Optical Instrumentation Engineers (SPIE) Conference Series, Society of Photo-Optical Instrumentation Engineers (SPIE) Conference Series 8148, Sept. 2011.

[73] R. K. Komanduri, K. F. Lawler, and M. J. Escuti, "Multi-twist retarders: broadband retardation control using self-aligning reactive liquid crystal layers," Opt. Express 21, pp. 404-420, Jan 2013.

[74] C. Oh and M. J. Escuti, "Achromatic diffraction from polarization gratings with high efficiency," Opt. Lett. 33, pp. 2287-2289, Oct 2008.

[75] M. N. Miskiewicz and M. J. Escuti 2014.

[76] J. S. Tyo, C. F. LaCasse, and B. M. Ratliff, "Total elimination of sampling errors in polarization imagery obtained with integrated microgrid polarimeters," Opt. Lett. 34, pp. 3187-3189, Oct 2009.

[77] D. A. LeMaster and K. Hirakawa, "Improved microgrid arrangement for integrated imaging polarimeters," Opt. Lett. 39, pp. 1811-1814, Apr 2014.

[78] H. Lin and A. Versteegh, "VisIRIS: a visible/IR imaging spectropolarimeter based on a birefringent fiber-optic image slicer," in Society of Photo-Optical Instrumentation Engineers (SPIE) Conference Series, Society of Photo-Optical Instrumentation Engineers (SPIE) Conference Series 6269, July 2006.

[79] P. Marconnet, L. Gendre, A. Foulonneau, and L. Bigué, "Cancellation of motion artifacts caused by a division-of-time polarimeter," in Society of Photo-Optical Instrumentation Engineers (SPIE) Conference Series, Society of Photo-Optical Instrumentation Engineers (SPIE) Conference Series 8160, Sept. 2011.

[80] S. Faisan, C. Heinrich, F. Rousseau, A. Lallement, and J. Zallat, "Joint filtering estimation of stokes vector images based on a nonlocal means approach," J. Opt. Soc. Am. A 29, pp. 2028-2037, Sep 2012.

[81] G. Anna, N. Bertaux, F. Galland, F. Goudail, and D. Dolfi, "Joint contrast optimization and object segmentation in active polarimetric images," Opt. Lett. 37, pp. 3321-3323, Aug 2012.

[82] J. S. Tyo, J. E. N. Pugh, and N. Engheta, "Colorimetric representations for use with polarization-difference imaging of objects in scattering media," J. Opt. Soc. Am. A 15, pp. 367-374, Feb 1998.

[83] F. Snik, "Measure aerosols with ispex on your smartphone," SPIE newsroom, 2013. 